\title{
Anomalous magnetophotoluminescence as a result of level repulsion in arrays of quantum dots.
}

\author{
E. V. Tsiper ${ }^{1}$, P. D. Wang ${ }^{2 *}$, J. L. Merz ${ }^{2}$, A. L. Efros ${ }^{1}$, S. Fafard ${ }^{3 \dagger}$, D. Leonard ${ }^{3 \ddagger}$, and \\ P. M. Petroff ${ }^{3}$ \\ ${ }^{1}$ Department of Physics, University of Utah, Salt Lake City, UT 84112 \\ ${ }^{2}$ Department of Electrical Engineering, University of Notre Dame, Notre Dame, IN 46556 \\ ${ }^{3}$ Center for Quantized Electronic Structures (QUEST), Materials Department, and Department \\ of Electrical and Computer Engineering University of California, Santa Barbara, CA 93106
}

(April 30, 2019) 


\begin{abstract}
Selectively excited photoluminescence (SPL) of an array of self-organized $\mathrm{In}_{0.5} \mathrm{Ga}_{0.5}$ As quantum dots has been measured in a magnetic field up to $11 \mathrm{~T}$. Anomalous magnetic field sensitivity of the SPL spectra has been observed under conditions for which the regular photoluminescence spectra is insensitive to the magnetic field due to large inhomogeneous broadening. The anomalous sensitivity is interpreted in terms of the repulsion of excited levels of the dots in a random potential. A theory presented to describe this phenomena is in excellent agreement with the experimental data. The data estimated the correlation in the positions of excited levels of the dots to be $94 \%$. The magnetic field dependence allows the determination of the reduced cyclotron effective mass in a dot. For our sample we have obtained $m_{e} m_{h} /\left(m_{e}+m_{h}\right)=0.034 m_{0}$. 71.35.+z, 78.55.-m, 78.55.Cr, 76.40.+b
\end{abstract}

Typeset using REVTEX 
An array of self-organized quantum dots (QDs) is a unique system consisting of very

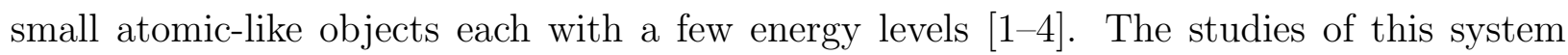
have shown the possibility to attain three-dimensional confinement of carriers within QDs. Such quantum dots are formed in highly strained semiconductor heterostructures by what is known as Stranski-Krastanow growth, where growth starts two-dimensionally, but after a certain critical thickness is reached, islands are formed spontaneously, and a thin wetting layer is left under the islands. In this process, the growth is interrupted immediately after the formation of the islands and before strain relaxation and misfit dislocations occur. Such insitu formation of $0 \mathrm{D}$ quantum dots results in high quality defect-free materials. In addition, the coherent islanding and strain effects can produce QDs with a size uniformity within $\pm 10 \%$ which is very promising for 0D quantum devices where the sharper density of states is exploited.

Photoluminescence (PL) spectrum of such an array has a broad line which is supposed to be mainly due to inhomogeneous broadening [5,6]. Photoluminescence excitation (PLE) and selectively-excited photoluminescence (SPL) reveal a fine structure. This fine structure has been interpreted by one of us [7] as a result of splitting of the excited levels in quantum dots due to violation of cylindrical symmetry of the dots by a random potential. Though the splitting is much less than the PL linewidth, it can be observed because of the effect of repulsion of energy levels inside a dot. Here we give an experimental proof of this point and a detailed study of the level repulsion in quantum dots by applying magnetic field which affects the splitting of excited levels.

We found an anomalous sensitivity of the SPL to a magnetic field under conditions for which the regular photoluminescence spectra is insensitive to the magnetic field due to large inhomogeneous broadening. We show that this sensitivity is a direct result of the level repulsion. A preliminary discussion of this effect has been given earlier $[8]$.

The dot layer studied here is pseudomorphically grown by MBE on (100) GaAs substrate, and the QDs are formed by the coherent relaxation into islands of a few monolayers (ML) of $\mathrm{In}_{0.5} \mathrm{Ga}_{0.5} \mathrm{As}$ between GaAs buffer and cap layers. The actual amount of indium incorporated 
in the dots can differ due to the complex dynamics of the adatoms during island formation. The growth and QD structural details have been reported earlier [9].

Fig. 1(a) shows PLE and regular PL spectra. Regular PL reveals a broad line with the FWHM $=57 \mathrm{meV}$. PLE spectra consists of significantly narrower lines. PL spectrum is redshifted with respect to PLE by about $80 \mathrm{meV}$. This shift occurs since the PLE experiment detects the light emitted only by the levels below the level excited by the pumping light.

The SPL spectra for different excitation energies are presented in Fig. 1(b). The complicated character of the SPL spectra were analyzed earlier [7]. For our purposes it is important that at some energy of excitation $\left(E_{e x}=1.3672 \mathrm{eV}\right)$ the SPL spectrum shows two symmetric peaks. At larger and smaller $E_{\text {ex }}$ these peaks become asymmetric.

For an intuitive picture it is helpful to assume that each dot has two optically active excited levels $E_{ \pm}$relatively close to each other (see Fig. 2). The dots are isolated from each other, so the light is emitted from the same dot it is absorbed to. The dots are excited into $E_{+}$or $E_{-}$and, after thermalization, emit light from $E_{0}$. The red shift mentioned above originates from the difference $E_{ \pm}-E_{0}$. In some dots the excitation energy $\hbar \omega_{e x}$ may be close either to $E_{-}$or to $E_{+}$. These two types of dots can be considered as two different subsets. These subsets should give two peaks in the SPL curve if we assume the correlation between the positions of $E_{ \pm}$and $E_{0}$. If this correlation is of such kind that the dots with larger $E_{ \pm}$have, in general, larger $E_{0}$, the excitation of $E_{+}$will cause the lower peak in SPL. This is a natural proposal and it corresponds to our experimental data (see Fig. 1(b)). At some energy of excitation there are the same amounts of dots in each subset. However, if the energy of excitation is shifted upward, the number of the dots which have the lowest excited level at this energy is smaller, so the intensity of the higher-energy peak decreases. Correspondingly, the low-energy peak disappears as the excitation energy decreases.

In our interpretation two close optically active excited levels originate from the doubly degenerate lowest excited level of a cylindrically symmetric dot. These levels are split by a random potential which may include a deviation of a dot shape from cylindrical symmetry. It is crucial that both the distance between the peaks and their width, as well as the linewidth 
of the nonselective PL have the same origin. They are determined by the random potential which splits degenerate levels and shifts randomly all energy levels in QDs.

At the excitation energy $E_{e x}=1.3672 \mathrm{eV}$, which gives two symmetric peaks in the SPL, we have studied the magnetic field dependence of the SPL spectrum. The results presented in Fig. 3 show anomalous sensitivity to the magnetic field. The relative intensity of the dip between two peaks decreases by $11 \%$ in the magnetic field of $2 \mathrm{~T}$. Note that the wide line of the regular (non-selective) PL is insensitive to a magnetic field up to 10T [10].

We show below that the two-level structure of SPL at zero field and its anomalous sensitivity to the magnetic field are both the results of the level repulsion.

For an axially symmetric dot two degenerate wave functions with an angular momentum $|m|$ have the form $\Psi_{m \pm}(\mathbf{r})=\psi_{m}(r) e^{ \pm i m \phi}$, where $\psi_{m}$ can be chosen real. For the first excited state $m=1$. The positions of energy levels $\epsilon_{ \pm}$, split and shifted by random potential and by magnetic field, can be obtained as the eigenvalues of the secular matrix

$$
\delta H=\left[\begin{array}{l}
u+\frac{\hbar \omega}{2}, x+i y \\
x-i y, u-\frac{\hbar \omega}{2}
\end{array}\right],
$$

where the matrix elements $u$ and $x+i y$ take random values in each quantum dot and are given by [7, 11]:

$$
\begin{aligned}
u & =\int d^{3} r V(\mathbf{r}) \psi_{1}^{2}(\mathbf{r}), \\
x+i y & =\int d^{3} r V(\mathbf{r}) \psi_{1}^{2}(\mathbf{r}) e^{2 i \phi} .
\end{aligned}
$$

Here $V(\mathbf{r})$ is arbitrary Gaussian random potential. It can be caused by alloy fluctuations. In principle, the same description is also valid in the case when the cylindrical symmetry is violated by strain field or shape of the dots [7].

The values $u, x$, and $y$ are real independent Gaussian random variables with equal standard deviations $\sigma_{1}$. The eigenvalues of $\delta H$ are $\epsilon_{ \pm}=u \pm \Delta$, where $\Delta$ is the splitting of the excited level given by

$$
\Delta=\sqrt{\Delta_{0}^{2}+(\hbar \omega / 2)^{2}},
$$


$\Delta_{0}=\sqrt{x^{2}+y^{2}}$ being the splitting in the absence of the magnetic field. Magnetic field provides an extra splitting of the excited level, which for an axially-symmetric dot is equal to the cyclotron energy $\hbar \omega$.

The distribution function for $\Delta$ is easy to calculate:

$$
F(\Delta)=\frac{2 \Delta}{\sigma_{1}^{2}} \exp \left(-\frac{\Delta^{2}-(\hbar \omega / 2)^{2}}{\sigma_{1}^{2}}\right) \theta\left(\Delta^{2}-(\hbar \omega / 2)^{2}\right),
$$

where $\theta$ is the step function. At zero magnetic field $F(0)=0$ due to the level repulsion. In a finite field $F(\Delta)=0$ in the region $|\Delta|<\hbar \omega / 2$ since the splitting cannot be less than $\hbar \omega$. Now we show that PLE and SPL are closely related to the function $F(\Delta)$.

To find lineshape for both PLE and SPL, one should calculate intensity as a function of two frequencies $I\left(E_{e x}, E_{\text {det }}\right)$. The PLE and SPL lineshapes can be obtained from this function by fixing corresponding variables. We assume that matrix elements are energy independent, so the intensity is proportional to the distribution function $P\left(\epsilon_{0}, \epsilon_{1}\right)$ where $\epsilon_{0}$ and $\epsilon_{1}$ are the energies of the lowest and the next excited states respectively. To simplify the notation we measure energies from their average values, $\epsilon_{\alpha}=E_{\alpha}-\overline{E_{\alpha}}$.

The lowest state $E_{0}$ with the wave function $\psi_{0}(r)$ is also shifted by random potential by

$$
\epsilon_{0}=\int d^{3} r V(\mathbf{r}) \psi_{0}^{2}(\mathbf{r})
$$

The shift $\epsilon_{0}$ is also a Gaussian random variable which is statistically independent of $x$ and $y$, however, in general, it is correlated with $u$.

The general expression for $P\left(\epsilon_{0}, \epsilon_{1}\right)$ has the form:

$$
\begin{aligned}
P\left(\epsilon_{0}, \epsilon_{1}\right) & =\sum_{ \pm} \iiint d u d x d y \delta\left(\epsilon_{1}-u \mp \sqrt{x^{2}+y^{2}+(\hbar \omega / 2)^{2}}\right) \\
& \times G_{2}\left(\epsilon_{0}, u ; \sigma_{0}, \sigma_{1}, \rho\right) G_{1}\left(x ; \sigma_{1} / \sqrt{2}\right) G_{1}\left(y ; \sigma_{1} / \sqrt{2}\right)
\end{aligned}
$$

Here $\sigma_{0}$ is the dispersion of $\epsilon_{0}$.

The matrix element $u$ determines the overall shift of the excited level due to the random potential. $G_{1}(\epsilon ; \sigma)$ is the normal distribution, $G_{1}(\epsilon ; \sigma)=\exp \left(-\epsilon^{2} / 2 \sigma^{2}\right) / \sigma \sqrt{2 \pi}$.

The general form of the two-variable normal distribution of the variables $\epsilon_{0}$ and $u$ which takes into account the correlation between them is 


$$
G_{2}\left(\epsilon_{0}, u ; \sigma_{0}, \sigma_{1}, \rho\right)=\frac{1}{2 \pi \sigma_{0} \sigma_{1} \sqrt{1-\rho^{2}}} \exp \left\{-\frac{1}{2\left(1-\rho^{2}\right)}\left[\frac{\epsilon_{0}^{2}}{\sigma_{0}^{2}}-2 \rho \frac{\epsilon_{0} u}{\sigma_{0} \sigma_{1}}+\frac{u^{2}}{\sigma_{1}^{2}}\right]\right\}
$$

Here $\rho$ is the correlation coefficient, $|\rho| \leq 1$.

Taking integrals in Eq. (7) one obtains

$$
P\left(\epsilon_{0}, \epsilon_{1}\right)=G_{1}\left(\epsilon_{0} ; \sigma_{0}\right) D_{\rho}\left(\epsilon_{1}-\epsilon_{0} \rho \frac{\sigma_{1}}{\sigma_{0}} ; \sigma_{1}\right)
$$

where the function $D_{\rho}(\epsilon ; \sigma)$ is defined by

$$
\begin{aligned}
D_{\rho}(\epsilon ; \sigma)= & \frac{1}{\sigma} \sum_{( \pm \epsilon)}\left\{\frac{\sqrt{\mu-1}}{\mu \sqrt{\pi}} \exp \left(-\frac{(\hbar \omega / 2-\epsilon)^{2}}{(\mu-1) \sigma^{2}}\right)+\right. \\
& \left.\frac{\epsilon}{\sigma \mu^{3 / 2}} \exp \left(\frac{\mu(\hbar \omega / 2)^{2}-\epsilon^{2}}{\mu \sigma^{2}}\right)\left[1-\operatorname{erf}\left(\frac{\mu \hbar \omega / 2-\epsilon}{\sigma \sqrt{\mu(\mu-1)}}\right)\right]\right\},
\end{aligned}
$$

where $\mu=3-2 \rho^{2}$. The function $P\left(\epsilon_{0}, \epsilon_{1}\right)$ as determined by Eqs. (9), (10) is the generalization of the function introduced in [7] for zero magnetic field. Eq. (10) yields Eq. (5) with $\epsilon=\Delta$ when $\rho \rightarrow 1$.

The effect of level repulsion manifests itself in SPL and in PLE in full scale if the overall shift of $E_{ \pm}$is proportional to the shift of $E_{0}$. This means $\rho=1$. In this case we get

$$
P\left(\epsilon_{0}, \epsilon_{1}\right)=\frac{1}{\sigma_{0} \sqrt{2 \pi}} \exp \left(-\frac{\epsilon_{0}^{2}}{2 \sigma_{0}^{2}}\right) F\left(\epsilon_{1}-\epsilon_{0} \frac{\sigma_{0}}{\sigma_{1}}\right)
$$

where $F$ is the distribution function of splittings given by Eq. (5).

The PLE lineshape can be obtained from Eq. (11) by fixing the detection energy $\epsilon_{0}$. One can see that it is just given by the function $F$. To get SPL lineshape one should fix $\epsilon_{1}$. The lineshape of the SPL also reproduces the features of the function $F$. Because of the level repulsion both SPL and PLE are zero when $\epsilon_{1}=\epsilon_{0} \sigma_{1} / \sigma_{0}$. In the magnetic field they are zero in the range $\left|\epsilon_{1}-\epsilon_{0} \sigma_{1} / \sigma_{0}\right|<\hbar \omega / 2$.

If the fluctuations of the lowest and the next excited states were uncorrelated, the structure will be substantially smeared. However, the correlation occurs to be strong. In the model of alloy disorder the correlation coefficient $\rho$ is calculated to be $\rho=0.795$ [7]. The experimental value we obtain below is $\rho=0.945$. This coefficient describes the correlation 
in positions of the lowest excited level $E_{0}$ and the next split excited level $E_{ \pm}$. The value of $\rho$ close to 1 suggests that a substantial part of the fluctuations originates from the change of the size of the dots which shifts energy levels proportionally. That is why such a tiny effect as the repulsion of split levels makes SPL spectra very sensitive to a relatively weak magnetic field.

Fig. 4 shows the relative peak position and the relative valley intensity as a function of magnetic field. Solid lines represent the best fit to the experimental data with Eqs. (9), (10). The fit has been performed in the following way. The parameters $\rho, \sigma_{0}$, and $\sigma_{1}$ can be determined from zero-field data. Two dimensionless parameters, $\rho$ and $\sigma_{0} / \sigma_{1}$, are obtained from the relative depth of the dip in SPL and the shift of PLE maxima with detection energy as provided by Eq. (9). This gives $\rho=0.945$ and $\sigma_{0} / \sigma_{1}=\rho /\left(d E_{P L E} / d \hbar \omega_{\text {det }}\right)=1.189$. The absolute value of $\sigma_{1}$ can be found from the relative peak position at zero field, which gives $\sigma_{1}=21.4 \mathrm{meV}$ and $\sigma_{0}=25.5 \mathrm{meV}$. To check the consistency, we can obtain $\sigma_{0}$ from the width of the non-selective PL in Fig. 1(a). Assuming the Gaussian shape of PL, the FWHM $=57 \mathrm{meV}$ gives $\sigma_{0}=57 /(2 \sqrt{2 \log 2})=24.2 \mathrm{meV}$.

With these parameters given, the magnetic-field dependence of both the relative depth of the dip and the relative peak position depend only on the effective mass. The value $m_{\mathrm{eff}}=0.034 m_{0}$ gives perfect fit for both quantities as shown in Fig. 4.

The value of the effective mass we obtained can be understood by taking into account that the cyclotron frequency that enters Eq. (1) contains the reduced effective mass for the electron and hole, $m_{\mathrm{eff}}=m_{e} m_{h} /\left(m_{e}+m_{h}\right)$. If we assume $m_{e}=m_{h}$, our result would imply $m_{e}=0.068 m_{0}$. On the other hand, if we take for the effective mass of the electron the value for bulk $\mathrm{Ga}_{0.5} \mathrm{In}_{0.5} \mathrm{As}, m_{e}=0.045$ [12], the value of $m_{\mathrm{eff}}=0.034 m_{0}$ would give for the mass of the hole $m_{h}=0.14 m_{0}$. The small value of the hole mass is natural taking into account the two-dimensional nature of the QDs. On the other hand, the effects of strain and confinement are believed to increase the effective mass of the carriers with respect to the bulk material [13, 14].

In conclusion, we have observed anomalous sensitivity of SPL to the magnetic field 
low enough to affect the regular PL spectrum. We interpret such sensitivity in terms of the repulsion of energy levels in QDs caused by a random potential. We present a theory which describes the phenomenon and shows excellent agreement with the experimental data. The data allows to determine the correlation in the positions of excited levels the reduced cyclotron effective mass for the carriers in the dots.

\section{ACKNOWLEDGMENTS}

A.L.E. acknowledges the support of UCSB, subcontract No. KK3017 of QUEST. 


\section{FIGURES}

FIG. 1. PL and PLE (a) and SPL (b) spectra of InGaAs QDs with different excitation and detection energies.

FIG. 2. An intuitive picture of relevant energy levels in QDs. Note that excitation into the higher level gives lower-energy feature in SPL

FIG. 3. SPL spectra under different magnetic fields. The excitation energy is $\hbar \omega_{e x}=1.3673 \mathrm{eV}$.

FIG. 4. The relative valley intensity (squares) and peak position (triangles) as a function of magnetic field. The solid lines represent the best fit by Eqs. (9), (10). The parameters used are $\rho=0.945, \sigma_{0}=25.5 \mathrm{meV}, \sigma_{1}=21.4 \mathrm{meV}$, and $m_{\mathrm{eff}}=0.034 m_{0}$. 


\section{REFERENCES}

* current address: CoreTek, Inc., 25B Street, Burlington, MA 01803,

$\dagger \quad$ current address: Institute for Microstructural Sciences, National Research Council, Ottawa K1A 0R6, Canada,

‡ current address: Santa Barbara Research Center, Goleta, CA 93117.

[1] D. Leonard, M. Krishnamurthy, S. Fafard, J.L. Merz, and P.M. Petroff, J. Vac. Sci. and Technol. B 12, 1063 (1994).

[2] J.M. Moison, F. Houzay, F. Barthe, L. Leprince, E. Andre, and O. Vatel, Appl. Phys. Lett. 64, 196 (1994).

[3] J.M. Marzin, J.M. Gerard, A. Izrael, D. Barrier, and G. Bastard, Phys. Rev. Lett. 73, 716 (1994).

[4] M. Grundmann, J. Christen, N.N. Ledentsov, J. Bohrer, D. Bimberg, S.S. Ruvimov, P. Werner, U. Richter, U. Gosele, J. Heydenreich, V.M. Ustinov, A. Yu. Egorov, A.E. Zhukov, P.S. Kop'ev and Zh.I. Alferov, Phys. Rev. Lett. 74, 4043 (1995).

[5] S. Fafard, D. Leonard, J.L. Merz, and P.M. Petroff, Appl. Phys. Lett. 65, 1388 (1994).

[6] P.D. Wang, J.L. Merz, G.Medeiros-Ribeiro, S. Fafard, P.M. Petroff, H. Akiyama and H. Sakaki, Superl. and Micros. Dec (1996)

[7] E.V. Tsiper, Phys. Rev. B 54, 1959 (1996).

[8] E.V. Tsiper and A.L. Efros, 1996 March Meeting Abstract; E.V. Tsiper and A.L. Efros, Abstract for the Workshop on Optical Properties of Mesoscopic Semiconductor Structures, Snowbird, May 7-10, 1996; E.V. Tsiper and A.L. Efros, Proc. of 38th Electronic Materials Conference, Santa Barbara, June 26-28, 1996.

[9] R. Leon, S. Fafard, D. Leonard, J.L. Merz, and P.M. Petroff, Appl. Phys. Lett. 67, 521 (1995). 
[10] P.D. Wang, J.L. Merz, S. Fafard, R. Leon, D. Leonard, G. Medeiros-Ribeiro, M. Oestreich, P.M. Petroff, K. Uchida, N. Miura, H. Akiyama and H. Sakaki, Phys. Rev. B 53, 16458 (1996).

[11] A.L. Efros and M.E. Raikh, Effect of Composition Disorder on the Electronic Properties of Semiconducting Mixed Crystals, in: Optical Properties of Mixed Crystals ed. by R.J. Elliot and I.P. Ipatova, Elsevier, 1988.

[12] Numerical Data and Functional Relationships in Science and Technology, edited by O. Madelung, Landolt-Börnstein, New Series, Group III, Vol. 17, part a (Springer-Verlag, Berlin, 1982).

[13] M.A. Cusack, P.R. Briddon and M. Jaros, Phys. Rev. B 54, R2300 (1996).

[14] R. People and S.K. Sputz, Phys. Rev. B 41, 8431 (1990). 

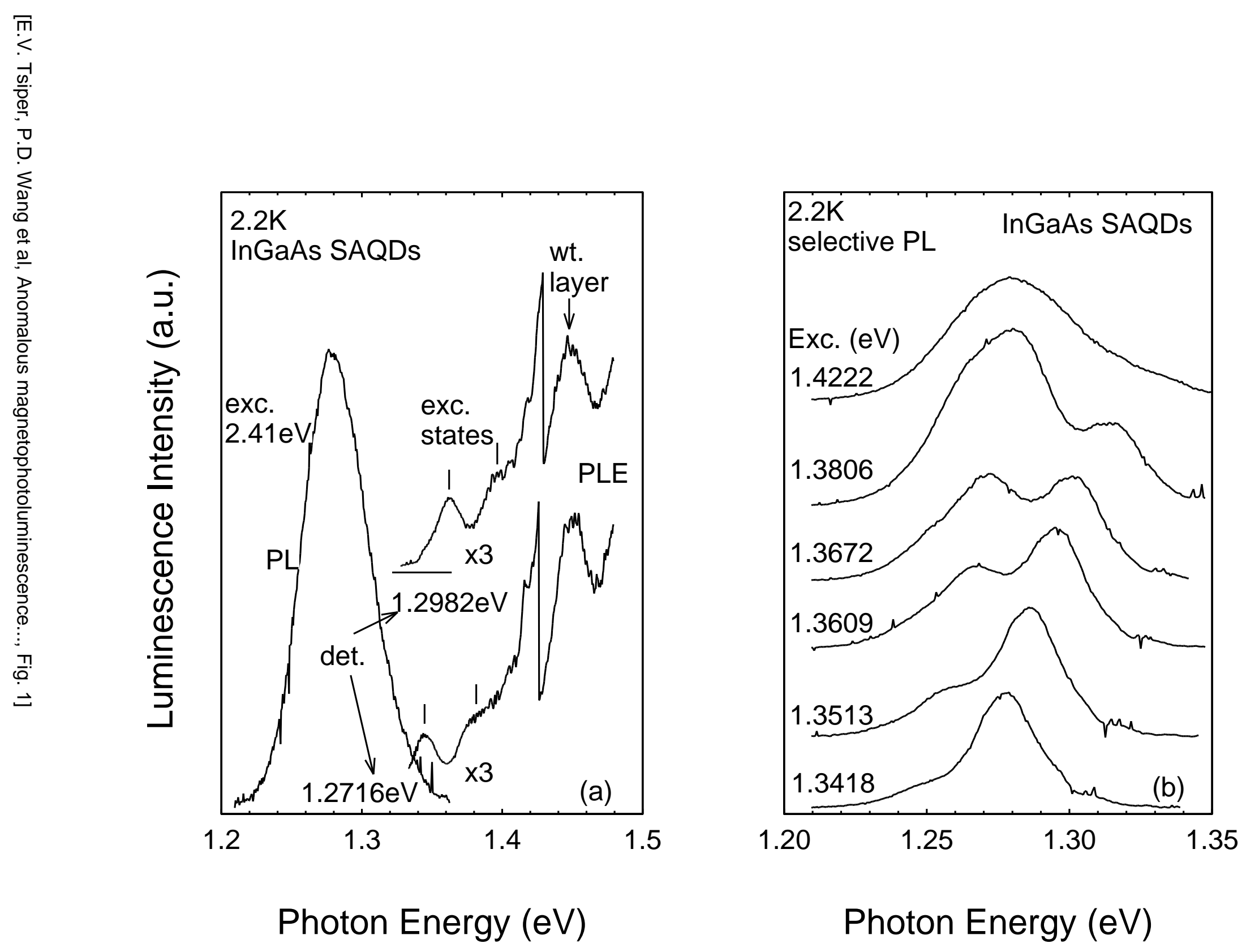


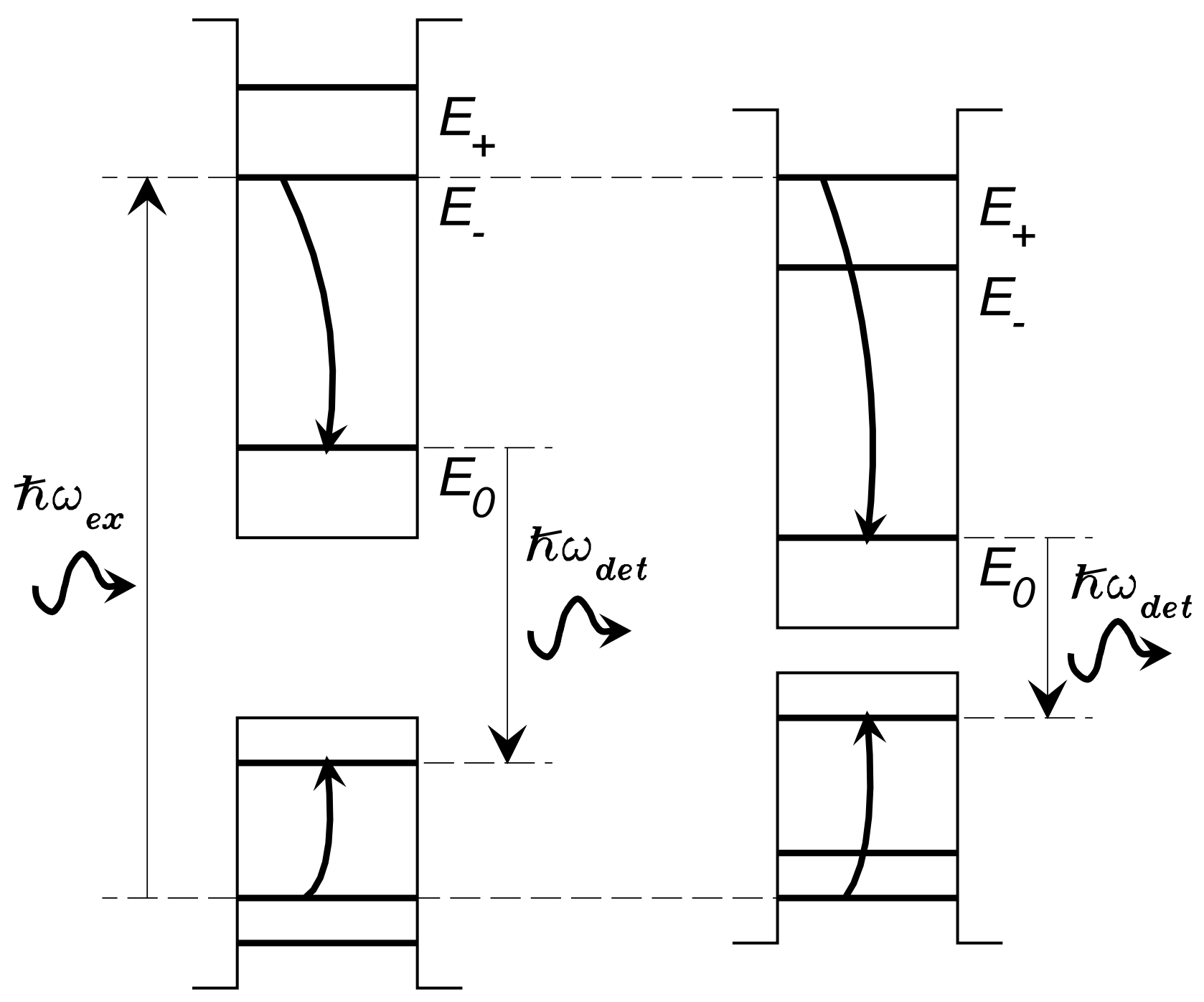




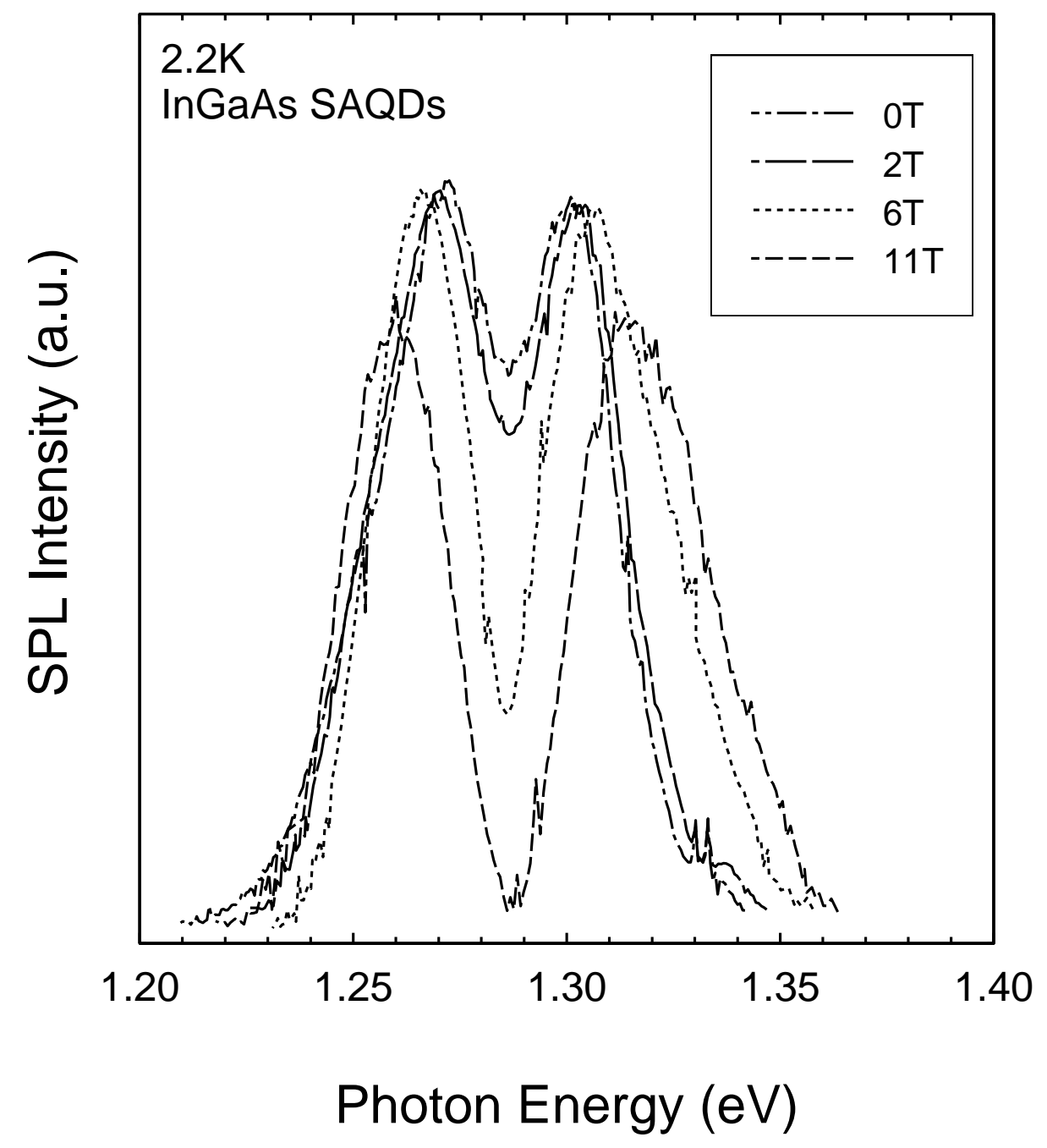




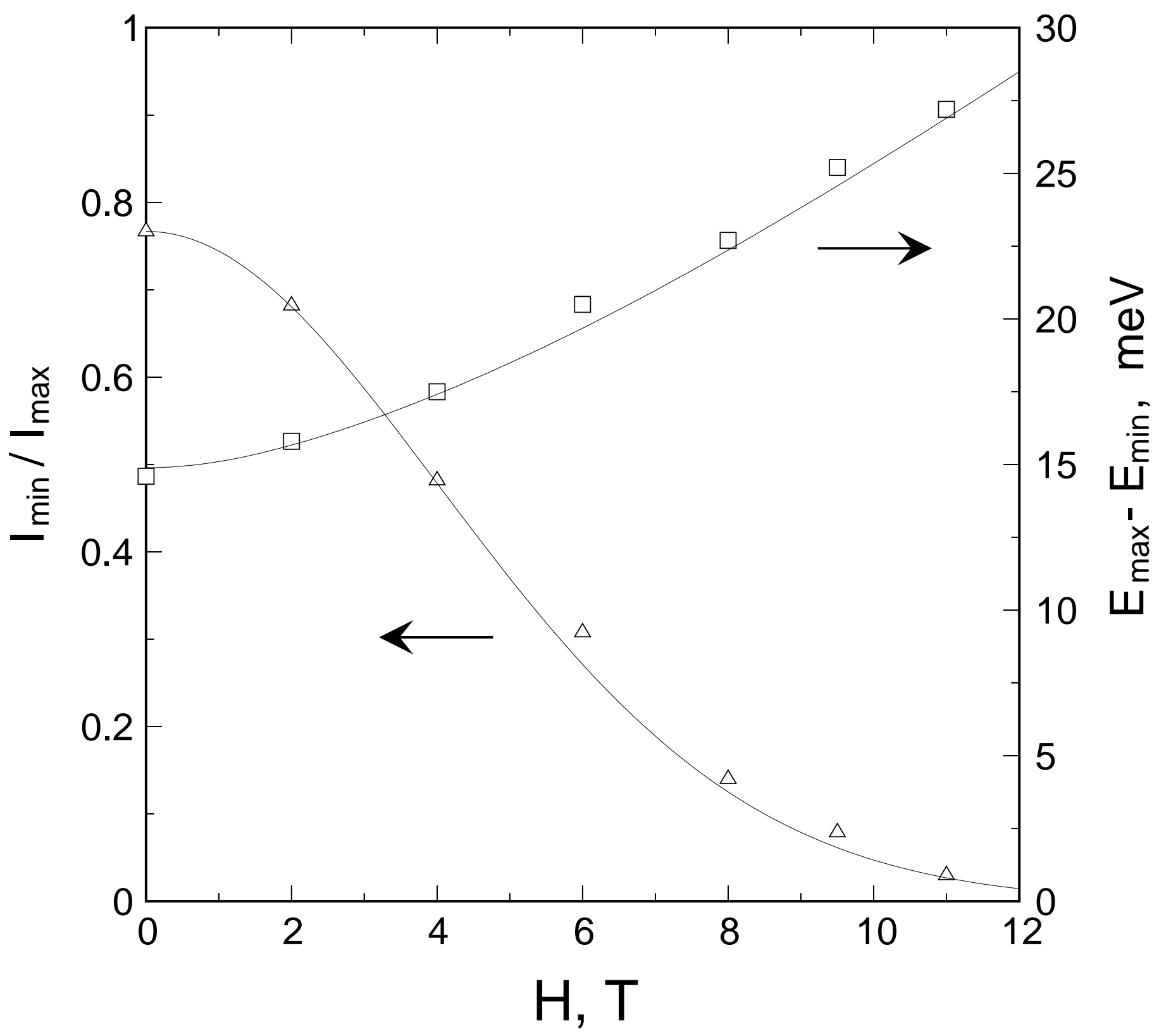

[E.V. Tsiper, P.D. Wang et al, Anomalous magnetophotoluminescence..., Fig. 4] 